\title{
Propagation du Lyssavirus chez les petits mammifères (Muridae et Soricidae) dans la Préfecture de Kindia, République de Guinée (Basse Guinée)
}

\author{
Namory KEITA ${ }^{1 *}$, Raphael DORE ${ }^{2}$, Aboubacar Hady TOURE ${ }^{2}$, Kerfalla KOUROUMA ${ }^{2}$ et \\ Mamadou Cellou BALDE ${ }^{2}$
}

${ }^{I}$ Faculté des Sciences, Département de Biologie, Université de Kindia, BP: 212 République de Guinée. ${ }^{2}$ Institut De Recherche En Biologie Appliquée De Guinée-Kindia, BP:146 République de Guinée.

*Auteurs correspondant ;E-mail: keitanamory13@gmail.com, raphaelzahoui@gmail.com, Tel: (+224)620-978-508.

Received: 03-05-2021

Accepted: 22-06-2021

Published: 30-06-2021

\section{RESUME}

L'étude porte sur la propagation du Lyssavirus chez les petits mammifères dans la Préfecture de Kindia (Basse Guinée) a été réalisée entre avril 2015 et mars 2016 dans les différents biotopes (Maisons habitées, ForêtsBuissons, Champs agricoles, Entrepôts et Berges) et qui a pour objectif: Contribuée à l'amélioration de l'état de santé de la population. Pendant cette période d'étude, 412 rongeurs repartis en 12 espèces ont été capturées à savoir le rat d'herbe-Arvicanthis rufinus, les rats-Cricetomys gambianus, les rats-Crocidurinae spp., les souris rayées-Lemniscomys striatus, Lophuromys ansorgei, Lophuromys sikapusi, le rat à mamelles multiplesMastomys spp., souris de maison-Mus musculus, Pelomys fallax, le rat de forêt-Praomys rostratus, le rat noirRattus rattus et le Malacomys edyardsi spp. Les plus répandus sont les Mastomys, Rattus rattus, Mus musculus et Cricetomys gambianus. En saison sèche, le nombre de rongeurs capturés dans la préfecture de Kindia a atteint $292(71 \%)$ contre $120(29 \%)$ rongeurs en saison pluvieuse. Pour des fins de recherches virologiques, le cerveau de 221 rongeurs a été prélevé et analysé parmi eux, 18 (8\%) ont été trouvé porteurs de l'ARN de Lyssavirus. (C) 2021 International Formulae Group. All rights reserved.

Mots clés : Rongeurs, Propagation, Lyssavirus, Réservoirs, Ecologie, Kindia.

\section{Spread of Lyssavirus among small mammals (Muridae and Soricidae) in Kindia prefecture, Republic of Guinea (Lower Guinea)}

\begin{abstract}
The study focuses on the spread of Lyssavirus among small mammals in the Prefecture of Kindia (Lower Guinea) was carried out between April 2015 and March 2016 in the different biotopes (inhabited houses, ForestsBushes, Agricultural fields, Warehouses and Banks) and which has for objective: Contributed to the improvement of the state of health of the population. During this period, 412 rodents were caught in 12 species including the grass rat-Arvicanthis rufinus, the Cricetomys gambianus-rats, the Crocidurinae spp rats, striped mice Lemniscomys striatus, Lophuromys ansorgei, Lophuromys sikapusi, the multiple-teat rat-Mastomys spp., house mouse-Mus musculus, Pelomys fallax, forest rat-Praomys rostratus, black rat-Rattus rattus, and Malacomys
\end{abstract}


edwardsi spp. The most common are: Mastomys spp., Rattus rattus, Mus musculus and Cricetomys gambianus. In the dry season, the number of rodents caught in the prefecture of Kindia reached 292 (71\%) against $120(29 \%)$ rodents caught during the rainy season. For virological research purposes, the brain of 221 rodents was collected and analyzed among them 18 rodents (8\%) were found to carry Lyssavirus RNA.

(C) 2021 International Formulae Group. All rights reserved.

Keywords: Rodents, Propagation, Lyssavirus, Reservoirs, Ecology, Kindia.

\section{INTRODUCTION}

Le territoire de la République de Guinée, de par son écosystème riche et varié, renferme d'innombrables espèces végétales et animales parmi lesquelles on rencontre beaucoup de mammifères.

En Afrique, 6 des 14 espèces de Lyssavirus circulent (Hayman et al., 2016). D'après l'OMS, la rage est un problème de santé publique pertinent dans la plupart des pays africains où le risque d'infection humaine est élevé (OMS. 2013). Chaque année en Afrique, jusqu'à 24000 cas de décès de rage humaine a été signalés (Hernachudha et al., 2013). La principale source d'infection humaine est le chien enragé, dans la faune, le virus de la rage circule parmi les carnivores et les ongulés (Warrell 2010). Parmi les autres Lyssavirus africains, les agents pathogènes humains sont les virus Mokola et Ouvenhage. Plus tard, le virus a été isolé chez les rongeurs non vaccinés et vaccinés contre la rage des chiens et des chats, ainsi que sur des humains (Kgaladi et al., 2013). Le Lyssavirus Duvenhage a été isolé à plusieurs reprises dans le cerveau d'humains décédés d'une maladie similaire à la rage et de chauves-souris insectivores cliniquement saines (Paweska et al., 2006). Le Lyssavirus de la chauve-souris de Lagos a été isolé uniquement à partir d'animaux tels que les chauves-souris elles-mêmes, les chats, les chiens et les mangoustes (Kuzmin et al., 2008). Pour les Lyssavirus de la chauvesouris lkoma et Shimoni, le réservoir naturel et la pathogénicité pour l'homme n'ont pas été établis (Banyard et al., 2014).

En République de Guinée, la propagation de deux types de Lyssavirus (virus de la rage et de la chauve-souris) a été précédemment confirmée (Kuzmin et al., 2008; Youla et al., 2014).
Dans cette classe zoologique, certains sont considérés comme des réservoirs de maladies infectieuses zoonotiques. En effet, les trois quarts des maladies infectieuses émergentes ont une origine zoonotique. On peut, par exemple, citer la maladie due au virus Ebola qui a sévit en Afrique de l'Ouest, ou la maladie de Lyme qui se développe en Europe et en Amérique du Nord, et dont la prévalence a augmenté fortement ces dernières années (Eden-Emerging diseases/Home (Internet). Cité 31 janv 2014).

Les coccidioses sont reconnues comme une dominante pathologique parmi les parasitoses du lapin. Elles sont les plus dangereuses chez cette espèce animale et constituent un problème sanitaire majeur de l'élevage cunicole partout où cet élevage est pratiqué (Akpo et al., 2011).

Plusieurs études menées en Afrique sur le virus (Owoade et al., 2004; Hassan 2004; Mardassi et al., 2004; Kasanga et al., 2013; Jembery et al., 2014), ont rapportées que les souches virales du sérotype I peuvent être classées dans trois génotypes: génotype des souches virulentes classiques, celui des souches variantes et celui des souches hypervirulentes.

Une implication des rongeurs dans la transmission du virus au sein des exploitations où le fumier est stocké à côté des bâtiments d'élevage a été rapportée au Sénégal (Badji et al., 2017). Une telle pratique est aussi observée au Bénin par Boko et al. (2015) et sur les plantes utilisées dans le traitement des pathologies d'origine bactérienne (Ogni et al., 2016).

Les rongeurs sont des animaux sensibles aux conditions environnementales de leur milieu: une modification de ces conditions peut entraîner la pullulation d'une espèce. Par 
exemple, on a observé des pullulations de rongeurs champêtres suite à des changements dans l'utilisation des terres agricoles. D'autre part, certaines espèces de rongeurs vivent à proximité de l'homme, voire dans les habitats humains en tant que nuisibles, mais aussi, depuis quelques années, en tant qu'animaux de compagnie. Cette proximité avec l'homme et l'urbanisation croissante de la planète inquiètent, car ces espèces sont porteuses de maladies transmissibles à l'homme dont l'incidence peut augmenter significativement en fonction de l'évolution des conditions de vie de ces animaux et de l'homme (EdenEmerging diseases/Home (Internet). Cité 31 janv 2014).

S'agissant de la Préfecture de Kindia (Basse Guinée), région des plaines côtières, on ne dispose pas beaucoup de renseignements sur la répartition et l'abondance de toutes les espèces, en dehors de rapports scientifiques publiés en 2000 par le département de Zoologie Médicale de l'IRBAG.

Le genre Lyssavirus fait partie des 13 genres dans la famille des rhabdovirus (Rhabdoviridae) et contient 14 espèces (Virus Taxonomy. 2015). Selon la classification proposée par Hayman et ses co-auteurs, les Lyssavirus sont génétiquement hétérogènes et se divisent en plusieurs groupes phylogénétiques (Hayman et al., 2016). Le groupe I de Lyssavirus comprend le virus de la rage, les Lyssavirus européens, eurasiens et australiens de la chauve-souris et le virus africain Duvenhage. Le groupe II se compose uniquement de Lyssavirus africains-Mokola, Lagos et Shimoni; le groupe phylogénétique III comprend les Lyssavirus de la chauve-souris du Caucase occidental, lkoma et Lleida. L'objectif de ce présent travail, est de contribuer à l'amélioration de l'état de santé de la population.

\section{MATERIEL ET METHODES}

Notre étude, prévue pour trois ans de terrain, s'est déroulée de janvier 2015 à décembre 2018 et chaque site a été prospecté trois fois par an durant l'étude mais la capture des petits mammifères a été réalisée entre avril
2015 et mars 2016 en utilisant une méthode standardisée quantitative.

Au cours de nos travaux de recherche portant sur la Propagation du Lyssavirus chez les petits mammifères (Muridae et Soricidae) dans la Préfecture de Kindia, République de Guinée (Basse Guinée), nous avons capturé 412 rongeurs répartis en 12 espèces.

Les animaux ont été capturés dans la banlieue de Kindia et le centre administratif de la ville de Kindia. Pour l'étude, des homogénats de cerveau à $10 \%$ ont été préparés en utilisant une solution stérile de chlorure de sodium à $0,9 \%$, qui ont été centrifugés pendant $2 \mathrm{~min}$ à $3000 \mathrm{tr} / \mathrm{min}$. Ensuite, 100 microns litres de surnageant ont été ajoutés à 300 microns litres de solution de lyse du kit "AmpliPrime RI BO-Prep" (Interlab Service, Russie) et incubés pendant 20 minutes à $65^{\circ} \mathrm{C}$.

L'isolement d'ARN a été effectué en utilisant des réactifs pour l'isolement d'ARN/ADN à partir de matériel clinique "AmpliPrime RIBO-Prep" (InterlabService, Russie) selon les instructions données par le fabricant.

La construction de l'ADNc a été réalisée en utilisant un kit "Reverta-L" (1nterlab Service, Russie) en tenant compte des instructions du fabricant. Pour identifier le matériel génétique du Lyssavirus, la PCR a été réalisée en deux cycles en utilisant des amorces spécifiques au genre complémentaires de la région du gène de la nucléoprotéine $\mathrm{N}$. Le kit de réactifs qPCR-mix-HS (Evrogen, Russie) a été utilisé pour la PCR selon les instructions du fabricant. Protocole PCR: $95{ }^{\circ} \mathrm{C}-2$ minutes $(1$ cycle), $94{ }^{\circ} \mathrm{C}$ - 5 secondes, $56{ }^{\circ} \mathrm{C}-10$ secondes, $72{ }^{\circ} \mathrm{C}-30$ secondes (40 cycles) et $72{ }^{\circ} \mathrm{C}-7$ minutes. Le séquençage des fragments de PCR a été réalisé sur un analyseur génétique automatique ABI Prism 310 Genetic Analysis (ABI, USA) complet avec le module logiciel Combined Applications, en utilisant le kit de séquençage de cycle BigDye Terminator v3.1 (ABI, USA). Toutes les études ont été menées au Centre de recherche russo-guinéen en épidémiologie et Prévention des maladies infectieuses dans la préfecture de Kindia Basse Guinée), République de Guinée. 


\section{RESULTATS}

Pendant notre étude, nous avons capturé 412 petits mammifères entre avril 2015 et mars 2016 à l'aide du système Guero (Tapettes), S.H. Sherman, Atrarat et Harmless to man

Du Tableau 1, il ressort que les investigations zoologiques dans les différents biotopes de la préfecture de Kindia ont montré que l'abondance ou la prédominance des rongeurs au niveau des berges et des Champs agricoles étaient de même 28,00\% suivi de 25,00\% dans les Entrepôts, 14,00\% dans les Forêts-Buissons. La plus faible capture a été remarquée dans les maisons d'habitation avec 5,00\%.

L'analyse du Tableau 2 indique que la densité de la distribution des espèces capturées par biotope suit un ordre décroissant: Berges, Champs-agricoles, Entrepôts, Forets-Buissons et Maisonshabitées biotope suit un ordre décroissant: Berges, Champs-agricoles, Entrepôts, ForetsBuissons et Maisons-habitées.

Les espèces les plus fréquemment rencontrées en fonction de leurs biotopes par ordre décroissant sont les: Mastomys spp., Rattus rattus, Mus musculus, Cricetomys gambianus, Crocidura spp., Lemniscomys striatus, Praomys rostratus. Les moins représentées sont Arvicanthis rufinus, Pelomys fallax, Malacomys edvardsi spp.. Enfin, les plus faiblement représentées sont les Lophuromys ansorgei et Lophuromys sikapusi.

Le Tableau 3 montre la répartition des espèces capturées dans la Préfecture de Kindia. En tenant compte de celles faiblement représentées (Lophuromys ansorgei et Lophuromys sikapusi) avec $1(0,24 \%)$ pour chacun des cas à celles fortement représentées ou dominantes (Mastomys spp.) avec 167 $(40,53 \%)$. Nous estimons que l'abondance de la nourriture et de la température ont été les causes favorables pour la pullulation des rongeurs.

Du Tableau 4, on constate que l'ARN de Lyssavirus (Rabdovirudae) a été détecté au niveau de 18/221 rongeurs analysés à la PCR. Tous les essais positifs ont été passés au test pour la détection du virus de la rage mais l'ARN du virus de la rage n'a été détecté dans aucun prélèvement. Cependant, il faut signaler aussi qu'aucun représentant des Lophuomys sikapusi et Lemniscomys striatus n'est passé au test de Lyssavirus.

Tableau 1: Distribution des rongeurs capturés par biotope.

\begin{tabular}{lcc}
\hline Biotope & Nombre & $\%$ \\
\hline Maisons habitées & 20 & 5,00 \\
\hline Forets-Buissons & 59 & 14,00 \\
\hline Champs agricoles & 114 & 28,00 \\
\hline Entrepôts & 102 & 25,00 \\
\hline Berges & 117 & 28,00 \\
\hline Total & 412 & 100 \\
\hline
\end{tabular}


Tableau 2: Distribution des espèces capturées par biotope.

\begin{tabular}{|c|c|c|c|c|c|c|}
\hline No & Espèces & $\begin{array}{l}\text { Maisons } \\
\text { habitées }\end{array}$ & $\begin{array}{c}\text { Forets- } \\
\text { Buissons }\end{array}$ & $\begin{array}{l}\text { Champs } \\
\text { agricoles }\end{array}$ & Entrepôts & Berges \\
\hline 1 & Arvicanthis rufinus, Temminck, 1853 & - & - & - & - & 2 \\
\hline 2 & Cricetomys gambianus, Waterhouse, 1840 & - & 9 & 2 & 4 & 4 \\
\hline 3 & Crocidurinae spp., Wagler, 1832 & - & 4 & 1 & 1 & 5 \\
\hline 4 & Lemniscomys striatus, Linnaeus, 1758 & - & 6 & 3 & - & 1 \\
\hline 5 & Lophuromys ansorgei, Winton, 1896 & - & - & - & - & 1 \\
\hline 6 & Lophuromys sikapusi, Temminck, 1853 & - & - & 1 & - & - \\
\hline 7 & Mastomys spp., Thomas, 1915 & - & 31 & 64 & 29 & 43 \\
\hline 8 & Mus musculus Linnaeus, 1758 & 15 & - & - & 7 & - \\
\hline 9 & Pelomys fallax, Peters, 1852 & - & - & - & - & 2 \\
\hline 10 & Praomys rostratus, Thomas, 1905 & - & 5 & - & - & 4 \\
\hline 11 & Rattus rattus, Linnaeus, 1758 & 5 & 4 & 43 & 61 & 53 \\
\hline 12 & $\begin{array}{l}\text { Malacomys edvardsi ssp, Rochebrune, } \\
1885\end{array}$ & - & - & - & - & 2 \\
\hline Total & 12 & 20 & 59 & 114 & 102 & 117 \\
\hline
\end{tabular}

Tableau 3: Espèces capturées au cours des enquêtes.

\begin{tabular}{clccc}
\hline No & Espèces & Nombre & \% & $\begin{array}{c}\text { Indice de } \\
\text { dominance } \\
\text { en \% }\end{array}$ \\
\hline 1 & Mastomys spp., Thomas, 1915 & 167 & 40,53 & 40,53 \\
\hline 2 & Rattus rattus, Linnaeus, 1758 & 166 & 40,29 & 40,29 \\
\hline 3 & Mus musculus Linnaeus, 1758 & 22 & 5,33 & 5,33 \\
\hline 4 & Cricetomys gambianus, Waterhouse, 1840 & 19 & 4,61 & 4,61 \\
\hline 5 & Crocidurinae spp., Wagler, 1832 & 11 & 2,66 & 2,66 \\
\hline 6 & Lemniscomys striatus, Linnaeus, 1758 & 10 & 2,42 & 2,42 \\
\hline 7 & Praomys rostratus, Thomas, 1905 & 9 & 2,18 & 2,18 \\
\hline 8 & Arvicanthis rufinus, Temminck, 1853 & 2 & 0,48 & 0,48 \\
\hline 9 & Pelomys fallax, Peters, 1852 & 2 & 0,48 & 0,48 \\
\hline 10 & Malacomys edvardsi ssp, Rochebrune, 1885 & 2 & 0,48 & 0,48 \\
\hline 11 & Lophuromys ansorgei, Winton, 1896 & 1 & 0,24 & 0,24 \\
\hline 12 & Lophuromys sikapusi, Temminck, 1853 & 1 & 0,24 & 0,24 \\
\hline Total & & 412 & 100 & 100 \\
\hline Pourcentage total & & 100 & 100 \\
\hline
\end{tabular}


Tableau 4: Analyse par PCR du cerveau des rongeurs (détection de l'ARN de Lyssavirus et le virus de la rage).

\begin{tabular}{|c|c|c|c|c|c|c|}
\hline \multirow[t]{2}{*}{ No } & \multirow[t]{2}{*}{ Espèces } & \multirow{2}{*}{$\begin{array}{l}\text { Nombre de } \\
\text { rongeurs } \\
\text { Examinés }\end{array}$} & \multicolumn{2}{|c|}{ Positif } & \multicolumn{2}{|c|}{ Négatif } \\
\hline & & & Nombre & $\%$ & Nombre & $\%$ \\
\hline 1 & Arvicanthis rufinus, Temminck, 1853 & 1 & - & - & 1 & 0,45 \\
\hline 2 & $\begin{array}{l}\text { Cricetomys gambianus, Waterhouse, } \\
1840\end{array}$ & 1 & - & - & 1 & 0,45 \\
\hline 3 & Crocidurinae spp., Wagler, 1832 & 6 & 2 & 1,00 & 4 & 1,80 \\
\hline 4 & Lemniscomys striatus, Linnaeus, 1758 & - & - & - & - & - \\
\hline 5 & Lophuromys ansorgei, Winton, 1896 & 2 & - & - & 2 & 0,90 \\
\hline 6 & Lophuromys sikapusi, Temminck, 1853 & - & - & - & - & - \\
\hline 7 & Mastomys spp., Thomas, 1915 & 78 & 12 & 5,42 & 66 & 29,86 \\
\hline 8 & Mus musculus Linnaeus, 1758 & 9 & 1 & 0,45 & 8 & 3,61 \\
\hline 9 & Pelomys fallax, Peters, 1852 & 1 & - & - & 1 & 0,45 \\
\hline 10 & Praomys rostratus, Thomas, 1905 & 4 & - & - & 4 & 1,80 \\
\hline 11 & Rattus rattus, Linnaeus, 1758 & 117 & 3 & 1,35 & 114 & 51,58 \\
\hline 12 & $\begin{array}{l}\text { Malacomys edvardsi ssp, Rochebrune, } \\
1885\end{array}$ & 2 & - & - & 2 & 0,9 \\
\hline Total & 12 & 221 & 18 & 8,00 & 203 & 92,00 \\
\hline$\%$ & & 100 & 8,00 & 8,00 & 92,00 & 92,00 \\
\hline
\end{tabular}

\section{DISCUSSION}

Ces 412 petits mammifères capturés lors de nos travaux appartiennent à deux ordres (Muridae et Soricidae) et les plus représentatifs étaient les : Mastomys spp. 167, Rattus rattus 166, Mus musculus spp. 22, Cricetomys gambianus 19, Crocidura spp. 11, Lemniscomys striatus 10. Par contre, les moins rencontrées sont : Praomys rostratus 9, Pelomys fallax 2, Arvicanthis rufinus 2, Malacomys edvardsi ssp. 2, Lophuromys sikapusi 1 et Lophuromys ansorgei 1. Ces espèces représentent l'essentiel de la faune des rongeurs, susceptibles de jouer un rôle épidémiologique ou de prédateurs pour les cultures et les récoltes.

Les résultats de l'analyse d'échantillons de cerveau animal pour la présence d'ARN de Lyssavirus sont présentés dans le Tableau 4. L'ARN des Lyssavirus a été trouvé chez des animaux de tous les ordres. Tous les échantillons positifs sont testés pour le virus de la rage. L'ARN du virus de la rage n'a été détecté dans aucun des échantillons.

Les animaux infectés ont été capturés à proximité des lieux de résidence permanente des humains (maisons, fermes avicoles, plantations, jardins potagers), ce qui offre un potentiel d'infection par les Lyssavirus chez l'homme. Apartir d'un pool d'échantillons de cerveau de rat noir ( $R$. rattus), capturé dans une ferme avicole, a été identifié un fragment (9088-9207 b) du gène de la protéine de matrice M des Lyssavirus.

Sur le continent africain, 6 des 14 espèces de Lyssavirus circulent: virus de la rage, chauve-souris de Lagos, Duvenhage, Mokola, lkoma et chauve-souris Shimoni (Hayman et al., 2016) et en 2017 dans la même préfecture, lors de nos travaux, l'analyse de 221 cerveaux de petits mammifères (rongeurs) au laboratoire mobile de l'IRBAG/CREMS a détecté l'ARN de Lyssavirus dans 18 cas positifs sur 221 prélèvements de cerveaux. 
Selon l'OMS, la rage est un problème de santé publique pertinent dans la plupart des pays africains où le risque d'infection humaine est élevé (OMS. 2013).

Auparavant, il y avait des rapports sur l'isolement du virus Mokola au Nigeria, en Afrique du Sud, au Cameroun, en République centrafricaine, au Zimbabwe et en Éthiopie (Kgaladi et al., 2013). L'isolement de ce virus à partir de chiens et de chats vaccinés contre la rage, et l'étude in vitro de neutralisation croisée avec des anticorps antirabiques d'autres Lyssavirus confirment le faible effet protecteur de la vaccination antirabique contre le virus Mokola (Boldbaatar et al., 2009). Ainsi, l'analyse d'échantillons de cerveau des représentants des quatre ordres petits mammifères capturés dans la banlieue de Kindia (République de Guinée), a montré la présence d'ARN du Lyssavirus dans 8,7\% des échantillons. On a identifié et séquencé un court fragment d'ARN génomique du virus Mokola dans des échantillons de rats noirs. L'analyse phylogénétique a permis de faire l'hypothèse que le fragment séquencé du génome appartient au nouveau génotype du virus Mokola. Malgré le fait que tous les échantillons positifs à la PCR n'ont pas été séquencés, les données obtenues permettent d'exclure la présence d'ARN du virus rabique dans les échantillons positifs. A cet égard, il semble pertinent une étude plus approfondie sur l'épidémiologie moléculaire du virus Mokola, isolement des Lyssavirus pour une étude plus détaillée et l'identification d'autres espèces de Lyssavirus circulant en Afrique de l'Ouest. Il est également fondamental d'évaluer le rôle possible des Lyssavirus, et du Mokola virus en particulier, dans le développement de pathologies neurologiques sévères chez les résidents de Guinée.

\section{Conclusion}

Les germes pathogènes liés à ces rongeurs se propagent d'hôtes en hôtes, d'espèces en espèces lorsque les conditions optimales sont réunies. Ces conditions sont donc liées aux modifications des écosystèmes. L'évolution des populations de rongeurs sauvages est très dépendante de l'utilisation de l'environnement par l'homme. Une modification dans les modes de cultures, des terrains laissés en jachères, sont autant de conditions favorables à la pullulation de rongeurs champêtres. Dans nos différents biotopes ou sites d'étude, les populations de rongeurs commensaux, généralement abondantes, connaissent beaucoup de fluctuations saisonnières que les populations sauvages. Cela est dû en partie à une disponibilité alimentaire permanente et une reproduction continue. A l'extérieur, elle est saisonnière et commence généralement au milieu de l'hivernage (saison des pluies) où elle est dépendante des cultures. A l'opposé, il y a beaucoup plus d'espèces pendant la saison sèche où les fortes densités de rongeurs se retrouvent dans nos Maisons d'habitations, Forets et Buissons, Champs, Entrepôts et aux berges comme le montre ce présent résultat. Cependant, la découverte de Pelomys fallax suscite de discussions au niveau des chercheurs du Centre Guinéo-russe; pour eux, c'est une sous espèce de Praomys car l'espèce Pelomys fallax tend à disparaitre sur le territoire guinéen. Il faut aussi ajouter que Cricetomys gambianus ne doit plus être classé dans la Famille des Muridae mais plutôt dans la Famille des Nesomyidae, sous-Famille des Cricetomyinae.

\section{CONFLITS D'INTERETS}

Les auteurs déclarent qu'il n'y a pas de conflits d'intérêts pour cet article.

\section{CONTRIBUTIONS DES AUTEURS}

NK est l'auteur principal de ce travail et a participé à toutes les étapes de sa réalisation. Les contributions des auteurs comme RD, AHT, KK et MCB ont été immenses ; ils ont facilité la correction mais aussi contribué pour le suivi et la publication du présent article.

\section{REMERCIEMENTS}

Nos remerciements vont à l'endroit des Chercheurs Russes du Centre de Recherche en Epidémiologie et des Soins Médicaux (CREMES), des Chercheurs de l'Institut de Recherche en Biologie Appliquée de Guinée (IRBAG) et ceux de l'Université de Kindia 
(UK). Nos remerciements vont aussi à l'endroit des relecteurs anonymes dont leurs critiques et suggestions nous ont permis d'améliorer cet article.

\section{REFERENCES}

Akpo Y, Kpodékon MT, Djago Y, Licois D, Youssao IAK. 2011. Evaluation de l'innocuité de Eimeria magna et de Eimeria media issues du Bénin en vue de leur utilisation comme souches vaccinales. Int. J. Biol. Chem. Sci., 5(4): 1682-1687.

DOI:

http://dx.doi.org/10.4314/ijbcs.v5i4.30

Badji A, Lo MM, Alambedji RB. 2017. Facteurs de risque liés à l'apparition de la maladie de Gumboro en aviculture semiintensive au Sénégal. International Journal of Biological and Chemical Sciences, 11(4): 1515-1558. DOI: http://dx.doi.org/10.4314/ijbcs.v11i4.182

Banyard AC, Evans JS, Luo TR, Fooks AR. 2014. Lyssaviruses and Bats. Emergence and Zoonotic Threat Viruses, 6: 29742990. DOI: 10.3390/v6082974.

Boko MA, Dougnon TV, Bankolé HS, Dougnon TJ, Ahouangninou C, Cledjo P, Soumanou M. 2015. Pratiques d'élevage avicole au Sud-Bénin (Afrique de l'Ouest) et impacts sur l'hygiène des fumiers produits. International Journal of Biological and Chemical Sciences, 9(6): 2740-2753.

DOI: http://dx.doi.org/10.4314/ijbcs.v9i6.18.

Boldbaatar B, Inoue S, Sugiura N, Noguchi A, Orbina JR, Demetria C, Miranda ME, Yamada A. 2009. Rapid detection of rabies virus by reverse transcription loopmediated isothermal amplification. Jpn. J. Infect. Dis. 2009; 62 (3): 1876191.

Eden-Emerging diseases/Home (Internet). Cité 31 janv. 2014. Disponible sur: https://www.eden-f p6project.net/emerging-diseases.

Hassan MK. 2004. Very virulent Infectious Bursal Disease Virus in Egypt: epidemiology, isolation and immunogenicity of classic vaccine. Veterinary Research, 28: 347-350. DOI: https://dx.doi.org/10.4314/ijbcs.v13i1.1.
Hayman DT, Fooks AR, Marston DA, Garcia RIC. 2016. The global Phylogeography of Lyssaviruses-Challenging the 'Out of Africa' Hypothesis. PLoS. Negl. Trop., 10 (12): e0005266. DOI: 10.1371/journal.pntd.0005266.

Hernachudha T, Ugolini G, Wacharapluesadee S, Sungkarat W, Shuangshoti S, Laothamatas J. 2013. Human rabies: neuropathogenesis, diagnosis andmanagement. Lancet Neurol., 12: 498-513. DOI: $10.1016 / 51474-$ 4422(13)70038-3.

IRBAG. Rapport Scientifique. 2000. Activité du Virus Lassa chez les Petits Mammifères Capturés en Guinée (19962000), pp. 28-34. Kindia, Mars 2001.

Jembery S, Stacey EL, Fekadu K, Robert MC, Esayas G, Haileleul N, Kassahun Gelagay A. 2014. Genetic characterisation of infectious bursal disease virus isolates in Ethiopia. Acta Tropica, 130: 39-43. DOI: https://dx.doi.org/10.4314/ijbcs.v13i1.1.

Kasanga CJ, Yamaguchi T, Munangandu HM, Ohya K, Fukushi H. 2013. Genomic sequence of an infectious bursal disease virus isolate from Zambia: classical attenuated segment B reassortment in nature with existing very virulent segment A. Archives of Virology, 158: 685-689. DOI: 10.1007/s00705-012$1531-4$

Kgaladi J, Wright N, Coertse J, Markotter W, Marston D, Fooks AR, Freuling CM, Muller TF, Sabeta CT, Nel LH. 2013. Diversity and Epidemiology of Mokola Viruse. PLos Negl.Trop., 7(10):e2511. DOI: 10.1371/journal.pntd.0002511.

Kuzmin IV, Niegoda M, Franka R, Agwanda B, Markotter W, Beagley JC, Urazova QY, Breiman RF, Rupprecht CE. 2008. Lagos Bat Virus in Kenya. I. Clin. Microbial., 46(4): 1451-1461. DOI: 10.1128/ICM.00016-08.

Mardassi H, Khabouchi N, Ghram A, Namouchi A, Karboul A. 2004. A Very Virulent Genotype of Infectious Bursal Disease Virus Predominantly Associated with Recurrent Infectious Bursal Disease Outbreaks in Tunisian Vaccinated Flocks. 
Avian Diseases, 48: 829-840. DOI: https://dx.doi.org/10.4314/ijbcs.v13i1.17

Ogni CA, Kpodekon M, Dougnon J, Dassou H, Goussanou JE, Boko C, Koutinhouin B, Youssao I, Akoegninou A. 2016. Dominant bacterial diseases in the extensive and semi-intensive animal breeding and their treatment method by ethnoveterinary medicine in Benin. $J$. App. Pharm. Sci., 6(04): 150-158.

Ogni CASW. 2016. Enquête ethnopharmacologique et efficacité des extraits de quelques plantes sur deux pathologies animales fréquentes au Bénin. Thèse de doctorat, Université d'Abomey-Calavi, Bénin, p. 215.

OMS. 2013. Global distribution of risk to humans of contracting rabies, 2013. [Cited 01.032017], URL: http://www.who.int/rabies/Global_ distribution_risk_humans_contracting rabies_2013.png?ua=1.

Owoade AA, Mulders MN, Kohnen J, Ammerlaan W, Muller CP. 2004. High sequence diversity in infectious Bursal disease virus serotype I in poultry and turkey suggests west-African origin of very virulent strains. Archives of Virology, 149: 653-672. DOI: https://dx.doi.org/10.4314/ijbcs.v13i1.17
Paweska JT, Blumberg IH, Liebenberg C, Hewlett RH, Grobbelaar AA, Leman PA, Croft IE, Nel IH, Nutt I, Swanepoel R. 2006. Fatal Human Infection with Rabiesrelated Duvenhage Virus, South Africa. Emerg. Infect. 12: 1965-1967. DOI: 10.3201/eid1212.060764.

Plan de développement Communal Horizon, 2015. Commune Urbaine de Kindia.

Plan de Développement des Collectivités Locales de la Préfecture de Kindia, Document provisoire, Juillet, 2008.

Virus Taxonomy. 2015. Release EC 47, London, UK, July 2015. [Oted 01.03.2017\}. URL: http://www.ictvonline.org/virusTaxonom y.asp.

Warrell M. 2010. Rabies and African bat Lyssavirus encephalitis and its prevention. Int. J. Antimicrob. Agents, 36(1): 547-52. DOI: 10.1016/j.ijantimicag.2010.06.021.

Youla AS, Traore FA, Sako FB, Feda RM, Emeric MA. 2014. Canine and human rabies in Conakry: epidemiology and preventive aspects. Bull. Soc. Pathol. Exot., $107(1)$ : $18-21 . \quad$ DOI: 10.1007/s13149-013-0321-x. 